\title{
TRANSFORMACIÓN PEDAGÓGICA MEDIADA POR TECNOLOGÍAS DE LA INFORMACIÓN Y LA COMUNICACIÓN (TIC)*
}

\author{
Miguel Garcés-Prettel ${ }^{1}$ \\ Rosmayra Ruiz-Cantilloº \\ David Martínez Ávila ${ }^{3}$
}

\begin{abstract}
RESUMEN
El objetivo de este artículo es fundamentar teóricamente el concepto de transformación pedagógica y su relación con las tecnologías de la información y la comunicación. El análisis epistemológico giró en torno a las posibles bases teóricas que sustentan el concepto, lo que implica en el contexto educativo desarrollar procesos de transformación pedagógica mediada por este tipo de tecnologías y las posibles formas de integrarlas en las dinámicas de enseñanza-aprendizaje para potenciar la interacción, la autonomía, el aprender a aprender, el trabajo colaborativo y la participación activa de los estudiantes en su formación académica.
\end{abstract}

\section{PALABRAS CLAVES}

Transformación pedagógica, aprendizaje, docencia, integración tecnológica.

\begin{abstract}
The goal of the present article is to theoretically support the concept of pedagogical transformation and its relation with information and communication technologies. The epistemological analysis revolves around the theoretical possible basis that support the concept, which means that in the education context there is a need to develop pedagogical transformation processes mediated by this kind of technologies and the possible ways of joining them in the teaching-learning dynamics in order to maximize the interaction, the autonomy, the learn to learn, the team work, and the active participation of the students in their academic training.
\end{abstract}

\section{KEYWORDS}

Pedagogic Transformation, Learning, Teaching, Technologic Integration

Depositado en agosto 15 de 2014, aprobado en octubre 31 de 2014.

* Este articulo forma parte del estado del arte (revisión teórica y de antecedentes) de una investigación patrocinada por la Universidad Tecnológica de Bolívar y el Departamento Administrativo de Ciencia, Tecnología e Innovación de Colombia (COLCIENCIAS) dentro del marco de la convocatoria 525 de 2011.

1 Magister en comunicación. estudiante del doctorado en comunicación, Universidad del Norte. Profesor Asociado Universidad Tecnológica de Bolívar. Email: mgarces@unitecnologica.edu.co

2 Comunicadora Social, Universidad Tecnológica de Bolívar. Joven investigadora, programa de Comunicación Social, Universidad Tecnológica de Bolívar. Email: rous.ruiz@gmail.com.

3 Profesor asistente, Universidad Tecnológica de Bolívar, Magíster en Educación. E-mail: djmartin@unitecnologica.edu.co 


\section{INTRODUCCIÓN}

El término Transformar según el Diccionario de la Real Academia Española, proviene del latín transformare que comunica la idea de hacer cambiar de forma a alguien o algo, o en su defecto, hacer mudar de porte o de costumbres a alguien. Podría entenderse entonces, que éste término aplicado al ámbito pedagógico presupone cambios favorables en el modo de concebir y desarrollar la práctica docente desde "algo" (el proceso de aprendizaje) con "alguien" (los actores educativos).

El proceso de aprendizaje dentro del sistema educativo está supeditado a diversos aspectos que inciden en su operatividad, entre estos: el factor institucional, que se configura a través del proyecto educativo institucional (PEI), la filosofía, los objetivos institucionales y el modelo de gestión educativa existente; el factor cultural que proviene del contexto histórico-social que permea a las instituciones educativas, junto con las características de la población que accede a este servicio; y el factor pedagógico que se alimenta de los anteriores, pero funciona con base en el currículo, los planes de curso y las dinámicas de aprendizaje que desarrollan docentes y estudiantes.

Todos estos factores son susceptibles de cambios continuos por las actuaciones y decisiones que toman los actores del sistema educativo (docentes, estudiantes, directivos, coordinadores, padres de familia) que aportan de forma distinta al proceso pedagógico a través de los roles que ejercen en calidad de gestores, dinamizadores, acompañantes, orientadores o aprendices.

Sin embargo, cuando se pregunta por el concepto de transformación pedagógica, no se hace referencia a cualquier cambio cotidiano que surge dentro del ámbito escolar. Garcés y Santoya (2011) dentro de sus reflexiones sobre reforma educativa mencionan este concepto para referirse a aquellos estilos de enseñanzaaprendizaje que generan innovaciones educativas que cambian las formas tradicionales de aprender centradas en la transmisión de información, por modelos pedagógicos que promueven el análisis, la comprensión, la interacción y la construcción social del conocimiento.
Bajo esta perspectiva, el concepto de transformación pedagógica implica para el docente innovar desde el modelo pedagógico y sus dimensiones centrales (las metas de formación, las metodologías, las relaciones, la comunicación entre los actores, la evaluación y los ritmos de aprendizaje) con el propósito de que el estudiante participe activamente de la gestión, generación y apropiación del conocimiento, permitiéndoles crecer en autonomía, responsabilidad, capacidad de expresión, pensamiento crítico-analítico, compromiso ético, y en todas aquellas competencias que contribuyen a la formación integral buscando que sean personas útiles a la sociedad.

Lograr esta finalidad exige consistencia entre cada una de esas dimensiones, pues no es coherente afirmar en las metas educativas, que el modelo pedagógico es constructivista, pero las metodologías y las relaciones que se implementan en las clases están reguladas exclusivamente por las sesiones magistrales, en donde el docente habla la mayor parte del tiempo y el estudiante actúa de forma pasiva y condicionada.

La pertinencia de estas dimensiones dentro de un modelo pedagógico transformador, radica en su compromiso por afinar los procesos de aprendizaje a partir de las características, necesidades y transformaciones del entorno social, político y productivo que permea a los actores, como también trabajando en el fortalecimiento de una cultura educativa que garantice los derechos y deberes fundamentales desde la formación para el saber ser, el saber hacer, el saber aprender y el saber convivir (UNESCO, 1996; 2001; 2008).

Paulo Freire, fue uno de los pedagogos en América Latina que habló de la educación para la transformación (pedagogía liberadora). Para éste pensador, la transformación pedagógica viene cuando el sistema educativo brinda posibilidades para que el educando se auto descubra y se conquiste reflexivamente como sujeto histórico constructor de su propio destino.

La perspectiva Freiriana parte del supuesto de que la transformación es posible en la medida que el sistema educativo parta de un modelo pe- 
dagógico contextualizado y aplicado, en donde se mire al sujeto en relación con su entorno sociocultural y político; en donde se potencien capacidades y posibilidades en los educandos para que enfrenten la vida y asuman consecuencias prácticas con lo que aprenden y deciden; en donde se ejercite una práctica pedagógica liberadora que reconozca los saberes que trae el estudiante y las posibilidades que tiene de trascender con lo que aprende (Freire, 1970; 1998; 1999).

Al debate de la transformación pedagógica se sumó desde la década del setenta el concepto de Tecnologías de la Información y la Comunicación (TIC), que surge en ese periodo como un intento de reconocer la importancia de los recursos tecnológicos (televisión, videograbadoras, radio, recursos audiovisuales, entre otros) para facilitar los procesos pedagógicos, pero ahora dicho concepto se ha ido enriqueciendo por la convergencia de la electrónica, las telecomunicaciones, la informática y la tecnología móvil dando paso a lo que hoy se conoce con el nombre de Nuevas Tecnologías.

En la actualidad los debates sobre transformación pedagógica incluyen necesaria e inevitablemente el papel de las TIC, de miras a mejorar o fortalecer las dinámicas pedagógicas dentro y fuera del aula. De allí el interés en este documento por entender las reflexiones y teorías que se han realizado sobre el tema, para una mejor comprensión de lo que implica desarrollar transformación en los escenarios educativos mediadas por este tipo de recursos.

\section{CONDICIONES PARA GENERAR TRANS- FORMACIÓN PEDAGÓGICA MEDIADA POR TIC}

Las TIC juegan un papel muy importante dentro los discursos sobre transformación pedagógica, ya que pueden contribuir al desarrollo de procesos de aprendizajes en donde los saberes se ejerciten buscando solucionar situaciones funcionales, complejas y cotidianas (Roegiers, 2004).

En materia de transformación pedagógica, otro aporte sustancial que pueden hacer los centros educativos con el apoyo de las TIC es generar estrategias que comprometan a los educandos con su propio aprendizaje, con su desarrollo hu- mano y con las transformaciones de las lógicas de poder al interior de la práctica educativa que debe propender por relaciones horizontales y por una convivencia centrada en el servicio, el respeto, la participación, el dialogo, el reconocimiento a la diversidad y al otro como legítimo otro (Rocha, 2005; Maturana, 1999). Al respecto Escontrela y Stojanovic plantean que:

Cada etapa ha tenido sus herramientas informáticas y también ha desarrollado modelos diferentes de utilización. En los inicios, décadas de los años sesenta y setenta, el modelo pedagógico de aplicación se basaba en la individualización del aprendizaje. En la década de los ochenta se empezó a hablar de la necesidad de promover el aprendizaje por descubrimiento y a destacar los beneficios del uso grupal. En la década de los noventa en adelante, el modelo cooperativo es el que recibió mayor atención debido, entre otras razones, al desarrollo de las comunicaciones (2004:5).

En el siglo XXI educar para una transformación pedagógica significa concebir el aprendizaje como un espacio de construcción social en donde se aprovechan las TIC con el fin de que el educando produzca, comparta y acceda a saberes, información y conocimientos relevantes que enriquezca su vivencia escolar.

Álvarez, Pérez y Suárez (2008) reafirman lo mismo, al considerar que una buena opción para la transformación pedagógica distanciada del enfoque tradicional son los nuevos modelos pedagógicos basados en la concepción constructivista y social del aprendizaje que les permite a los estudiantes aprender, poniendo en dialogo los conocimientos previos con los nuevos aprendizajes, organizando las interacciones sociales entre los actores educativos, y promoviendo un marco de aprendizaje en interacción permanente con el medio físico, social y tecnológico, con el objetivo de aplicar el conocimiento aprendido en la solución de situaciones concretas.

En el mismo orden de ideas, Cabero (2006) pone de manifiesto la importancia de movilizar estrategias diversas, incluyendo por supuesto las mediadas por TIC, para dar respuesta al cómo aprender y qué mecanismos se usarán 
para motivar y despertar el interés de los estudiantes en su formación, ya sea desde el uso individual de los sujetos con los materiales de estudio hasta la enseñanza para el uso grupal poniendo en acción el aprendizaje colaborativo.

La labor docente es vital para lograr procesos de transformación pedagógica viable y pertinente con el apoyo de las TIC, sin desconocer que se requieren condiciones básicas en donde el uso de estos recursos vaya más allá del interés por la cobertura y el acceso a la información. Precisamente la misma UNESCO $(1998 ; 2004)$ resalta esas condiciones básicas que hacen viable cualquier programa de formación docente soportado en la implementación de tecnologías para la información y la comunicación:

- Visión compartida: liderazgo proactivo y apoyo administrativo en todo el proceso de formación e integración de las TIC.

- Acceso: disposición de tecnologías, software y redes de telecomunicaciones para el trabajo docente.

- Educadores capacitados: la capacitación debe lograr que los docentes usen las TIC en entornos de aprendizaje.

- Desarrollo profesional: que los docentes tengan acceso continuo a instancias de desarrollo profesional para mantener actualizado en el uso de la tecnología en la enseñanza.

- Asistencia Técnica: es necesario que los educadores tenga a su disposición ayuda técnica para mantener y habilitar el uso adecuado de la tecnología.

- Estándares sobre contenido y recursos académicos: los docentes cuentan con los conocimientos sobre su materia, emplean metodologías adecuadas y cumplen con los estándares asociados al contenido. Esto es clave para poner las TIC al servicio de dichos contenidos y metodologías.

- Enseñanza centrada en el alumno: que no es otra cosa, que trabajar bajo un modelo de aprendizaje centrado en el estudiante.

- Evaluación: se realiza una evaluación permanente de la efectividad de la tecnología en el aprendizaje.

- Apoyo comunitario y político: La comunidad educativa y los socios de la institución proveen conocimientos, políticas de apoyo, financiamiento y estructuras de incentivo que posibilitan la implementación de las TIC en la educación.

- La promoción de nuevos roles en los docentes y los estudiantes para la generación de ambientes de aprendizaje usando las tecnologías como medios al servicio del desarrollo humano y de un proyecto educativo que valore la construcción social, cultural e interactiva del conocimiento.

En un escenario educativo que pretenda transformar la practica pedagógica convencional, el disponer de infraestructura tecnológica y recursos informáticos es imprescindible para desarrollar procesos de transformación pedagógica, "si se tiene en cuenta que uno de los mayores inconvenientes que suelen indicar los profesores a la hora de incorporar las TICs a la práctica educativa, es la falta de materiales educativos" (Cabero, 2004:7). Aun así, aunque los recursos tecnológicos juegan un papel importante, no lo hace ser lo transcendente dentro del proyecto educativo.

La trascendencia al parecer, tiene que ver con las formas innovadoras de usar o integrar las tecnologías para fortalecer el aprendizaje, brindando a los estudiantes nuevas posibilidades educativas en las distintas áreas del conocimiento o del currículo en general, en donde los sujetos más que compartir conocimientos e información, compartan significados, representaciones, valores, actividades y usos tecnológicos que despierten el deseo por aprender (Tenti, 2005).

La transformación pedagógica desde una mirada de la innovación educativa mediada por las TIC, tiene que ver con lo mencionado por Salinas (2004) y Martin-Laborda (2005), ambos coinciden en la necesidad que tienen las escuelas o centros educativos de realizar:

- Cambios en el proceso educativo: diversificando los espacios de formación más allá del aula, a través de una formación continua que permita la aparición de entornos virtuales de aprendizaje libres de las restricciones del tiempo y los lugares que exige la enseñanza presencial. 
- Cambios en el objeto de la enseñanza: por cuanto la formación no se reduce a labor de enseñar para memorizar contenidos, el desafío estriba en formar para el aprender a aprender.

- Cambios en los objetivos educativos: El nuevo objetivo consiste en dotar a los alumnos de las capacidades y de los conocimientos necesarios para vivir en esta sociedad de la información, en donde el estudiante debe producir conocimiento de valor, aprender a procesar la información, solucionar problemas y usar la información de manera responsable.

- Cambios en los centros escolares: gestionando acciones para la adquisición de infraestructura tecnológica, generación de políticas de integración de TIC, formación del profesorado para el uso de las tecnologías en la escuela y mayores espacios de participación de la comunidad educativa aprovechando las ventajas de las nuevas tecnologías.

- Cambios en las formas pedagógicas: en donde los docentes deben asumir nuevos roles centrados en facilitar un entorno favorable al aprendizaje basado en el diálogo y la confianza. Además debe actuar como un gestor de conocimiento y orientador del aprendizaje tanto a nivel general, como a nivel individual de cada alumno, con el fin de que el aprendiz aumente el interés por la materia estudiada, mejore la capacidad para resolver problemas, aprenda a trabajar en grupo, aprenda a comunicar sus ideas adquiriendo mayor confianza en sí mismos e incrementando su creatividad.

- Cambios en los contenidos didácticos: se debe posibilitar una mayor diversificación de fuentes de información, convergencias de lenguajes, mayor interactividad, generación de contenidos propios acorde al contexto y a las necesidades de los estudiantes.

Capacitar a los actores del proceso educativo, es un buen punto de partida para empezar a generar cambios en la cultura escolar y en los procesos pedagógicos que requieren mínimamente haber superado el analfabetismo tecnológico en la escuela. No obstante, la capacitación debe trascender el mero uso tecnológico y enfocar- se en la formación docente para el desarrollo de habilidades especiales que favorezcan el uso aplicado e integrado de las TIC en lo pedagógico y en lo curricular.

Diversos estudios internacionales como los realizados por Fowellin, Collins y Peter, Pouts y Riché, (Cit. en Garcés, 2009:4), han demostrado que el hecho de que los docentes aprendan a manejar los recursos informáticos y sus herramientas (software), no significa que sepan automáticamente cómo integrarlas y aplicarlas pedagógicamente, ya que todavía buena parte de los docentes en el mundo siguen usando las TIC por fuera de las clases, muy pocos son los que han logrado transformar pedagógicamente la labor educativa integrando o extendiendo los usos personales a sus actividades académicas.

\section{TRANSFORMACIÓN PEDAGÓGICA E INTEGRACIÓN DE LAS TIC}

La transformación pedagógica es posible lograrla desde dinámicas de integración tecnopedagógica, que focalicen las capacitaciones para cubrir necesidades formativas específicas que tengan los mismos docentes en sus esfuerzos por usar las TIC para mejorar los procesos de aprendizaje. Algunas de estas iniciativas de integración, han sido expuestas en los estudios realizados por Hernández y Quintero (2009), resaltándose la necesidad de que los docentes:

- Aprendan a seleccionar materiales curriculares,

- Adquieran competencias para saber evaluar recursos tecnológicos,

- Diseñen nuevos materiales curriculares,

- Utilicen la tecnología en los procesos formación,

- Diseñen situaciones de aprendizaje con TIC,

- Generen nuevos ambientes de aprendizaje mediados por TIC,

- Utilicen las TIC para evaluar,

- Apliquen las tecnologías para tratar la diversidad,

- Participen en proyectos de investigación e innovación

- Consideren el factor ético en esas iniciativas de uso e integración tecnológica en la escuela. 
Si los docentes se forman bien en el uso pedagógico de las TIC pueden alcanzar mejores niveles de integración de estos recursos en sus actividades académicas, en especial pueden lograr que sus estudiantes desarrollen habilidades y conocimientos esenciales como los descritos ampliamente por Roig (2002) que apuntan a la adquisición de competencias para:

- Saber utilizar las principales herramientas de Internet.

- Conocer las características básicas de los equipos.

- Diagnosticar qué información se necesita en cada caso.

- Saber encontrar la información.

- Saber resistir la tentación de dispersarse al navegar por Internet.

- Evaluar la calidad y la idoneidad de la información obtenida.

- Saber utilizar la información.

- Saber aprovechar las posibilidades de comunicación de Internet.

- Evaluar la eficacia y la eficiencia de la metodología empleada.

En todo este escenario que pretende entender lo que implica el concepto de transformación pedagógica mediada por TIC, han aparecido múltiples formas de integrar las TIC en el sistema educativo, tal vez las más comunes (que pueden darse gradual o alternadamente) son: la integración para la gestión administrativa, la integración para la gestión curricular o pedagógica, la integración para el diseño e implementación de proyectos pedagógicos.

\section{Integración de las TIC para la gestión ad- ministrativa}

La articulación de las TIC con lo administrativo y lo académico para mejorar integralmente la gestión organizacional de los centros educativos, sigue siendo un reto para muchas escuelas, pues normalmente los usos tecnológicos en esos escenarios se han reducido a la sistematización de información y a procedimientos que no trasciende lo técnico-operativo.

El informe sobre implantación y uso de las tecnologías en escuelas de educación primaria y secundaria de España 2005-2006, mostró precisamente que el uso más frecuente de las TIC en este campo está relacionado con la digitalización de procesos de matrícula, sistematización de expedientes, labores de comunicación con familias, profesores y otros centros educativos.

La integración de las TIC en la gestión administrativa aunque abarca los procesos de sistematización de información no lo agota, aquí lo esencial para hablar de transformación pedagógica estriba en la implementación de un modelo de gestión que ponga lo administrativo, lo logístico y lo financiero al servicio del fortalecimiento académico, con miras a diseñar e implementar políticas educativas para usos integrados y eficientes de las TIC en todas las áreas de la institución.

En otras palabras, la integración en esta perspectiva transformadora tiene como fin, poner lo estructural (incluyendo las plataformas tecnológicas y procedimientos administrativos) al servicio de las metas educativas y de los procesos pedagógicos, y no al revés.

\section{Integración de las TIC para la gestión curri- cular o pedagógica}

En lo concerniente a la relación entre transformación pedagógica e integración de las TIC para la gestión curricular, Sánchez (2003) enmarca este tipo de usos con el propósito de apoyar las disciplinas o contenidos curriculares haciendo del aprendizaje, los objetivos, y las estrategias de individualización y planeación educativa el centro del proceso y no los recursos tecnológicos. Este autor, definió un modelo de integración a partir del modelo de Fogarty, apuntando a tres vías: integración dentro de una disciplina, integración a través de las disciplinas, integración dentro de la mente del estudiante.

En este modelo se reconocen seis maneras de usar las TIC dentro del ámbito curricular:

- Forma anidada: se da cuando en un contenido específico de una asignatura los docentes logran que los estudiantes usen las TIC para desarrollar y ejercitar habilidades sociales y de pensamiento, además de aplicar aspectos puntuales del contenido tratado. 
- Forma tejida: los estudiantes usan las TIC para examinar conceptos e ideas, en temas relevantes de una asignatura que está tejido con otras disciplinas y contenidos.

- Forma enroscada: aquí se usan las TIC para "enroscar" habilidades sociales, de pensamiento y de inteligencias múltiples a través de varias disciplinas.

- Forma integrada: Las TIC son usadas como plataforma de apoyo para unir asignaturas en la búsqueda de superposiciones de conceptos e ideas.

- Inmerso: el estudiante filtra el contenido con apoyo de TIC y llega a estar inmerso en su propia experiencia.

- En red: el aprendiz filtra su aprendizaje con el uso de TIC, generando conexiones internas que lo llevan a interacciones externas con expertos de áreas afines.

En el mismo orden de ideas, Hooper \& Rieber (1995) señalan las distintas formas en que los docentes usan las TIC (algunos logran integrar estos recursos en los procesos pedagógicos, otros no las usan más allá del ámbito personal):

- Familiarización: los docentes aprenden a usar el computador y algunos programas, pero solo los utiliza en labores personales

- Utilización: los docentes usan las TIC en labores educativas de índole administrativa, sin articularlas a propósitos pedagógicos;

- Integración: usan las TIC para apoyar su labor docente en tareas y responsabilidades definidas dentro de los planes de curso;

- Reorientación: el docente por medio de las TIC facilita ambientes de aprendizaje para que sus estudiantes construyan saberes o conocimientos;

- Evolución: los docentes actualizan permanente su práctica pedagógica con base en las nuevas herramientas tecnológicas que van surgiendo y en los conocimientos sobre cómo aprenden las personas.

Por su parte Orjuela (2010) realiza una compilación importante de modelos de integración de TIC dentro del ámbito pedagógico que se han usado en diferentes contextos educativos, entre estos, resalta el modelo de la Fundación Gabriel
Piedrahita Uribe que consta de cinco ejes fundamentales (Dirección Institucional, Infraestructura en TIC, Coordinación y Docencia en TIC, Competencias Docentes para la Integración de las TIC, Recursos Digitales) que pasan por diferentes niveles antes de alcanzar su punto de madurez, en donde debe evidenciarse una transformación en los roles de los estudiantes y en la forma como se concibe la dinámica pedagógica dentro y fuera del aula:

- Nivel de preintegración: en donde el docente hace uso de las TIC para incrementar su productividad personal en labores comunicación, talleres y exámenes; almacenar y organizar información de estudiantes; mantener registro de calificaciones; comunicarse por correo electrónico, y utiliza Internet para localizar diversos recursos para las clases.

- Instrucción dirigida: El docente utiliza las TIC como herramienta de instrucción programada para que sus estudiantes se entrenen con prácticas, tutoriales y software de ejercicio.

- Integración básica: El docente emplea las TIC para elaborar mejores materiales para sus estudiantes; además, utiliza computador, software y video beam para enriquecer sus clases.

- Integración media: El docente solicita a los estudiantes utilizar diferentes herramientas informáticas y no informáticas para realizar trabajos de clase.

- Integración avanzada: El docente aprovecha las TIC y metodologías de aprendizaje activo para realizar proyectos de clase enfocados en el currículo, con el objeto de lograr mejoras en el aprendizaje de los estudiantes

Así mismo, Batista y Celso (2007) consideran que aplicar las TIC en las dinámicas de integración curricular o pedagógica significa concebirlas de forma transversal, bien sea como recursos didácticos, como objeto de estudio, como medios de producción, o como recursos para la expresión y la gestión de conocimientos que deben servir a objetivos pedagógicos que aporten a la construcción de identidades y a la promoción de espacios de comunicación, visibilización, formación y participación ciudadana desde la escuela. 


\section{Integración de las TIC para la gestión de proyectos pedagógicos}

Este tipo de integración pretende que el uso de las TIC genere transformación pedagógica en la medida en que se promuevan por fuera de las aulas iniciativas académicas de aplicación práctica del conocimiento, específicamente mediante la creación de proyectos pedagógicos.

Existen experiencias que demuestran como las TIC fortalecen mejor el accionar de los proyectos pedagógicos que tienen como objetivo: aplicar conocimientos para la solución de problemas de aprendizaje, reforzar saberes, o bien para que la comunidad educativa comprenda y asuma un compromiso transformador con las necesidades del contexto social, cultural, económico, político o ambiental en donde están inmersos los centros educativos.

Uno de los trabajos destacables de sistematización de experiencias de proyectos pedagógicos basados en TIC, fue el realizado por el Ministerio de Educación Nacional (MEN) de Colombia con poblaciones vulnerables. El MEN (2005) logró atraer 190 experiencias significativas de todo el país, de las cuales se seleccionaron 16 proyectos finalistas, 6 para grupos étnicos, 4 para poblaciones con necesidades educativas especiales, 5 para grupos en situación de conflicto y 1 para habitantes de frontera.

Dichas experiencias mostraron como las TIC bajo a esta modalidad, están contribuyendo significativamente a la promoción de la educación intercultural, el compromiso socioambiental, la afro-colombianidad, la etno-identidad, la convivencia para la paz, el desarrollo de una cultura artística y científica, la gestión empresarial, la intervención para mejorar problemas de autismo y déficit cognitivo, la multigradualidad y multiedad, entre otros temas.

Otro trabajo destacable en este mismo enfoque, es el realizado por Hernández et al (2011) que mencionan un sinnúmero de experiencias sobre proyectos pedagógicos mediados por TIC, y proponen una metodología especial para sistematizar rigurosamente estos procesos, partiendo de categorías como:
- El contexto socio-cultural de las comunidades,

- Los enfoques de enseñanza de las áreas de educación básica y media,

- Las posibilidades educativas que brindan las TIC para publicar información, desarrollar otras representaciones del mundo desde la ciencia y el arte, coordinar acciones educativas y negociar sentidos y significados dentro de los mismos procesos de aprendizaje.

Esta metodología es favorable para evaluar el alcance de la inserción de las TIC en las escuelas, pues detalla los indicadores que deben ser valorados a partir de esas categorías.

Todas estas experiencias mediadas por TIC son formas innovadoras de utilizar los recursos tecnológicos que dispone la escuela para relacionarlo con otros asuntos más allá de la clase tradicional, con el fin de resolver problemas del contexto social y escolar, y para fortalecer la cultura mediante la afirmación de los procesos identitarios y el desarrollo de procesos pedagógicos que potencien la creatividad de los estudiantes, reafirmándolos como seres pensantes, productivos, emprendedores y capaces.

\section{CONCLUSIONES}

Las transformaciones pedagógicas son más procesos que productos esperados, pues dependen no solo de la disposición del docente por integrar novedosamente las TIC para mejorar los procesos de aprendizaje, sino también de la escuela misma y de los gobiernos, que deben proveer capacitación y acceso a estos recursos tecnológicos que exigen para su aprovechamiento: disponer de una buena conectividad y de política claras para un uso educativo integrado y eficiente.

Aunque existen diferentes formas de concebir la transformación pedagógica mediada por el uso de las TIC, esta es posible lograrla cuando la educación es asumida como un espacio de construcción social que implica cambiar las viejas prácticas pedagógicas que miran a los educandos como simples receptores de información.

Para tal fin, se hace necesario incorporar modelos pedagógicos abiertos y flexibles que generen 
ambientes de aprendizaje, en donde el estudiante ejerza un rol más activo en su formación, encontrando con el apoyo de las TIC nuevas posibilidades de aprender y de insertarse en este entorno local y global en donde el acceso a la información, juega un papel decisivo en las decisiones diarias que toman los individuos y los grupos sociales, incluyendo los actores educativos.

Las transformaciones pedagógicas pueden partir de cambios en la cultura escolar y por supuesto en la cultura tecnológica. Con ambas cosas hay que trabajar, de tal manera que se diversifiquen y se amplíen los espacios de formación a la par de alcanzar una mayor cobertura tecnológica que posibilite erradicar el analfabetismo tecnológico en la escuela, y permita una mejor gestión administrativa que propenda por aprovechar al máximo las TIC, en aras de alcanzar las metas pedagógicas establecidas en el proyecto educativo institucional.

La transformación pedagógica implica comprender que las TIC son medios y no fines; son recursos y no el centro del proceso; facilitan los procesos educativos, más no garantizan en sí mismo su eficiencia. Bajo esta claridad, se hace imprescindible que los docentes no solo sepan usar las tecnologías informáticas, sino que aprendan a integrarlas en sus planes y actividades de curso, lo cual presupone no solo un cambio metodológico, sino un cambio de mirada en el modelo pedagógico y en el modo de concebir el rol de los docentes y estudiantes dentro y fuera del aula.

En este contexto de relaciones es determinante que los docentes reconozcan que los estudiantes son sujetos que traen saberes, que pueden asumir un compromiso ético con su propia vida y su propia formación, que pueden aprender a usar esos recursos tecnológicos para nutrir la clase compartiendo sus consultas, propuestas e indagaciones sobre el tema en cuestión. Este cambio de mirada puede potenciar en los estu- diantes la autonomía, el trabajo colaborativo, el interés por aprender y fortalecer el pensamiento crítico-reflexivo que son aspectos vitales en toda formación integral.

Los docentes en aras de lograr transformaciones pedagógicas viables, pueden integrar las TIC de diferentes formas y niveles, sin embargo no es suficiente el simple hecho de usar las TIC en las clases como soporte audiovisual o multimedial para la socialización de contenidos. Si solo las usan así, no dejaran de ser viejas metodologías con nuevas tecnologías, que cambian la forma del proceso educativo desde lo estético, pero en el fondo se sigue manteniendo el modelo transmisionista de antaño.

Estas reflexiones teóricas permiten dimensionar algunas formas de integración educativa innovadoras, en donde las TIC sirven de apoyo para desarrollar y ejercitar habilidades sociales y de pensamiento; para examinar conceptos e ideas sobre temas relevantes; para unir asignaturas en la búsqueda de superposiciones de ideas; para generar espacios de comunicación, visibilización, formación y participación ciudadana desde la escuela; para aplicar conocimientos en la solución de problemas de aprendizaje; para buscar información y fortalecer la cultura escolar mediante la afirmación de los procesos identitarios y el desarrollo de proyectos locales de emprendimiento.

Las transformaciones pedagógicas también se han hecho presente en diversas experiencias educativas mediadas por TIC, que develan como los docentes pueden hacer un uso eficiente de estos recursos para facilitar mejor las interacciones sociales, la comunicación, la aplicación práctica del conocimiento y el acceso a nuevas fuentes de información científica que actualicen la práctica educativa fundamentando mejor los contenidos y las estrategias en el aula.

\section{REFERENCIAS}

Álvarez, S., Pérez, A., \& Suárez, M. L. (2008). Hacia un enfoque de la educación por competencias. Principado de Asturias: Consejería de Educación y Ciencia. 
TRANSFORMACIÓN PEDAGÓGICA MEDIADA POR TECNOLOGÍAS DE LA INFORMACIÓN Y LA COMUNICACIÓN (TIC)

Batista, M. A., \& Celso, V. (2007). Tecnologías de la información y la comunicación en la escuela: trazos, claves y oportunidades para su integración pedagógica. Buenos Aires: Ministerio de Educación, Ciencia y Tecnología de la Nación.

Cabero Almenara, J. (2004). Cambios organizativos y administrativos para incorporación de las TICs a la formación: Medidas a adoptar. Revista Edutec de Tecnología Educativa n.18, 1-31.

Cabero Almenara, J. (Julio de 28 de 2006). Bases pedagógicas para la integración de las TICs en primaria y secundaria. Recuperado el 7 de Febrero de 2013, de Ponencia impartida en el II Congreso Internacional UNIVER - La Universidad en la sociedad de la Información : http://tecnologiaedu.us.es/cuestionario/bibliovir/Bases456.pdf

Escontrela Mao, R., \& Stojanovic Casas, L. (2004). La integración de las TIC en la educación: Apuntes para un modelo pedagógico pertinente. Revista Pedagógica Caracas, vol.25, n.74, 481-502.

Freire, P. (1970). Pedagogía del oprimido. New York: Herder \& Herder.

Freire, P. (1998). La educación como práctica de la libertad. Madrid: Siglo XXI.

Freire, P. (1999). La importancia de leer y el proceso de liberación. Madrid: Siglo XXI.

Garcés, M. E. (2009). Análisis comparativo del uso de las TIC en la educación primaria y secundaria de España con relación a otros países de Europa y Asia: El reto de conciliar lo tecnológico y lo pedagógico. Revista Q, vol.4, n.7, 7-8.

Garcés, M. E., \& Santoya, Y. E. (2011). Propuesta de reforma educativa superior en Colombia y Modelos Pedagógicos. Revista Cultural Unilibre, n.10, 78-85.

Hernández, A., \&, Quintero, A. (2009). La integración de las TIC en el currículo: necesidades formativas e interés del profesorado. REIFOP, vol.2, n.12, 103-119.

Hernández, U., Hernández, Y., Moreno, J., Anaya, S., \& Benavides, P. (2011). Los proyectos pedagógicos de Aula para la integración de las TIC. Popayan: Universidad del Cauca.

Hooper, S., \& Rieber, L. (1995). Teaching with technology. En A. Ornstein, Teaching: Theory into (págs. 154-170). Needham Heights: Allyn and Bacon.

Martin Laborda, R. (2005). Las nuevas tecnologías en la educación. Madrid: Fundación AUNA.

Maturana, H. (1999). Transformación en la convivencia. Santiago de Chile: Dolmen Ediciones.

MEN. (2005). Experiencias significativas para poblaciones vulnerables. Bogotá: Ministerio de Educación Nacional. Orjuela, D. (2010). Acercamiento a la integración curricular de las TIC. Praxis y saber, vol.1, n.2, 111-136.

Rocha, C. (2005). La comunicación y la participación. En CEDAL, Participación es reconocimiento (págs. 25-68). Bogotá: Centro de Comunicación Educativa Audiovisual.

Roegiers, X. (2004). Una pedagogía de la integración. Bruselas: Deboeck Universidad.

Roig, R. (2002). Las Nuevas Tecnologías aplicadas a la educación. Elementos para una articulación didáctica de las Tecnologías de la Información y la Comunicación. Alcoy: Marfíl.

Salinas, J. (2004). Innovación docente y uso de las TIC en la enseñanza universitaria. Revista de universidad y sociedad del conocimiento, vol.1, n.1, 1-16. 
Miguel Garcés Prettel, Rosmayra Ruiz Cantillo, David Martínez Ávila

Sánchez, J. (2003). Integración curricular de TICs: conceptos y modelos. Enfoques educacionales, vol.1, n.5, 51-65.

Tenti, F. (2005). La condición docente. Análisis comparado de la Argentina, Brasil, Perú y Uruguay. Buenos Aires: Siglo XXI.

UNESCO. (1996). La educación encierra un tesoro. Informe de la UNESCO de la Comisión Internacional sobre la educación para el siglo XXI, presidida por Jacques Delors. París: UNESCO.

UNESCO. (1998). Informe mundial sobre la educación: los docentes y la enseñanza en el mundo en mutación. Madrid: Organización de las Naciones Unidas para la Educación, la Ciencia y la Cultura .

UNESCO. (2001). Declaración de Cochabamba y recomendaciones sobre políticas educativas al inicio del siglo XXI. Recuperado el 23 de Enero de 2013, de Séptima Reunión del Comité Regional Intergubernamental del Proyecto Principal de Educación en América Latina y el Caribe realizada en Marzo de 2001: http://unesdoc.unesco.org/images/0012/001214/121485s.pdf

UNESCO. (2004). Las TICs en la formación docente. Guía de planificación. París: Organización de las Naciones Unidas para la Educación, la Ciencia y la Cultura.

UNESCO. (2008). Estándares de competencia en TIC para docentes. Londres: Organización de las Naciones Unidas para la Educación, la Ciencia y la Cultura. 
